\title{
Numerical Study of the Shielding Properties of Macroscopic Hybrid Ferromagnetic/ Superconductor Hollow Cylinders
}

\author{
Gregory P. Lousberg, Jean-Francois Fagnard, Marcel Ausloos, \\ Philippe Vanderbemden, and Benoît Vanderheyden, Member, IEEE
}

\begin{abstract}
We study the magnetic shielding properties of hybrid ferromagnetic/superconductor $(F / S)$ structures consisting of two coaxial cylinders, with one of each material. We use an axisymmetric finite-element model in which the electrical properties of the superconducting tube are modeled by a nonlinear $E-J$ power law with a magnetic-field-dependent critical current density whereas the magnetic properties of the ferromagnetic material take saturation into account. We study and compare the penetration of a uniform axial magnetic field in two cases: 1) a ferromagnetic tube placed inside a larger superconducting tube (Ferro-In configuration) and 2) a ferromagnetic tube placed outside the superconducting one (Ferro-Out configuration). In both cases, we assess how the ferromagnetic tube improves the shielding properties of the sole superconducting tube. The influence of the geometrical parameters of the ferromagnetic tube is also studied: It is shown that, upon an optimal choice of the geometrical parameters, the range of magnetic fields that are efficiently shielded by the hightemperature superconductor tube alone can be increased by a factor of up to 7 (2) in a Ferro-Out (Ferro-In) configuration. The optimal configuration uses a 1020 carbon steel with a thickness of $2 \mathbf{~ m m}$ and a height that is half that of the superconducting cylinder $(80 \mathrm{~mm})$.
\end{abstract}

Index Terms-Ferromagnetic/high-temperature superconductors (HTSs) hybrid structure, finite element, magnetic shield.

\section{INTRODUCTION}

$\mathbf{S}$ UPERCONDUCTIVITY and ferromagnetism are two seemingly different material properties, which, when allowed to interact, lead to numerous interesting physical effects at both the microscopic and macroscopic scales. At the microscopic scale, ferromagnetism and superconductivity are different electronic long-range orders that thermodynamically compete [1]. While singlet superconductivity and ferromagnetism are not likely to coexist in bulk compounds, coexisting correlations may be found in layered ferromagnetic/ superconductor $(\mathrm{F} / \mathrm{S})$ heterostructures [2] due to the proximity

Manuscript received August 28, 2009; revised November 9, 2009. First published December 8, 2009; current version published January 29, 2010. This paper was recommended by Associate Editor M. Parizh. The work of G. P. Lousberg was supported by the Fonds de la Recherche Scientifique (FRSFNRS) of Belgium under an FRS-FNRS fellowship.

G. P. Lousberg, M. Ausloos, P. Vanderbemden, and B. Vanderheyden are with the SUPRATECS, Department of Electrical Engineering and Computer Science, University of Liége, 4000 Liége, Belgium (e-mail: gregory.lousberg@ ulg.ac.be).

J.-F. Fagnard is with the CISS Department, Royal Military Academy, 1000 Brussels, Belgium, and also with the SUPRATECS, Department of Electrical Engineering and Computer Science, University of Liége, 4000 Liége, Belgium. Digital Object Identifier 10.1109/TASC.2009.2036855 effect, by which Cooper pairs are allowed to penetrate the ferromagnetic layer and induce superconducting correlations. This phenomenon is at the basis of spin-valve systems, where the proximity effect is used to control the electrical resistance of a trilayer $\mathrm{F} / \mathrm{S} / \mathrm{F}$ via an external magnetic field [3].

At the macroscopic scale, superconductors and ferromagnets also exhibit competing magnetic properties: In a given magnetic environment, the superconductor tends to expel the magnetic field lines from its volume by developing induced supercurrents [4]-[6]. By contrast, the ferromagnet, whose magnetic domains align with the external field, tends to concentrate the field lines inside its volume [7]. These opposite responses may simultaneously be exploited to improve the efficiency of high-temperature superconductors (HTSs) in a series of applications.

1) An increase in the electrical transport abilities in HTS tapes is achieved by placing ferromagnetic covers or ferromagnetic substrates of specific shapes [8]-[16]. In these F/S hybrid structures, the concentration of the magnetic field lines inside the ferromagnetic sheet attenuates the self-field in the wire, which consequently sustains a larger critical current with reduced AC losses.

2) Soft iron yokes are used in combination with bulk HTS in order to increase and stabilize the magnetic flux of HTS trapped flux magnets [17], [18]. These structures have promising applications in bulk HTS motors.

3) Type-II superconducting tubes are able to shield lowfrequency magnetic fields in a more efficient way than a ferromagnetic cylinder would do [5], [6]. The axial and transverse magnetic shielding properties of HTS cylinders may even be improved by covering the HTS tube with a ferromagnetic sheath [19]-[24].

In this paper, we focus on F/S coaxial tubes designed for magnetic shielding. Numerous studies were carried out on such systems [20]-[23]. Most studies were experimental and focused on the configuration where the ferromagnetic tube is placed outside the superconducting tube (the Ferro-Out configuration). The opposite configuration, where the ferromagnetic tube is placed inside the superconducting one (the Ferro-In configuration), is encountered in practice when the HTS material consists of a coated conductor deposited on a cylindrical magnetic metal, e.g., Ni [25]. Indeed, in that case, one may ask what effect the substrate may have on the magnetic response of the system and whether this effect depends on the side of the 
substrate that has been coated. To the best of our knowledge, the modeling of the Ferro-In configuration has never been considered in that context.

Other questions also remain open: How are the shielding properties of a hybrid F/S system influenced by the magnetic saturation of the ferromagnetic layer? How can the geometry of each separate layer be modified in order to optimize the shielding properties of the composite system?

Our work aims at investigating these questions on F/S hybrid magnetic shields by means of a numerical model. In the literature, several models with macroscopic F/S structures have been developed for calculating the field penetration and the $\mathrm{AC}$ losses in HTS tapes with ferromagnetic substrates or covers. They usually rely on finite-element formulation implemented on commercial software [8]-[10], [15]. In this study, we use the finite-element method (FEM) for calculating the penetration of a uniform axial magnetic field in a system comprising two coaxial tubes made of HTS and ferromagnetic materials. The model takes into account the nonlinear conductivity of the HTS, as well as the nonlinear magnetic permeability of the ferromagnetic material. It is implemented in the open-source solver GetDP [26], [27]. With the help of that model, we study and compare configurations where the ferromagnetic layer is placed outside (Ferro Out) or inside (Ferro In) the HTS tube. In particular, we study the influence of the saturation of the magnetic layer on the shielding efficiency of the hybrid structure and investigate whether this shielding efficiency can be optimized by proper choice of the geometrical parameters of the ferromagnetic layer.

This paper is organized as follows. The finite-element model is described in Section II. Section III is devoted to the simulation of the shielding properties of the HTS and of the ferromagnetic tubes used alone. The hybrid structures consisting of the superposition of $\mathrm{F} / \mathrm{S}$ tubes are studied in detail in Section IV. Section V summarizes our findings and presents the conclusions.

\section{MOdel FOR MACROSCOPIC F/S Structures}

We consider macroscopic F/S hybrid structures consisting of the superposition of two coaxial tubes of finite height, i.e., a ferromagnetic and an HTS one. The tubes are subjected to a magnetic field that is uniform and parallel to their axis. To reduce the computation load, the tubes are represented in a 2-D axisymmetric geometry. Fig. 1 shows a sketch of the simulation domain containing both tubes surrounded by vacuum. The simulation domain is limited by the revolution axis, the median plane of the tube (for symmetry reasons), and a quarter of a circle with radius $r_{\text {circle }}=20 r_{\text {int }}$, where $r_{\text {int }}$ denotes the inner radius of the HTS tube. The outer limit of the domain $r_{\text {circle }}$ was empirically fixed by trial and error in such a way that any small variation of $r_{\text {circle }}$ around the chosen value leads to a negligible variation of the magnetic flux density calculated in the vicinity of the tubes.

We use a formulation of the Maxwell equations in terms of the vector potential $\mathbf{A}$. In the axisymmetric geometry defined in Fig. 1, the vector potential has a single nonvanishing component oriented along the azimuthal direction $\mathbf{A}=A \mathbf{e}_{\theta}$. The

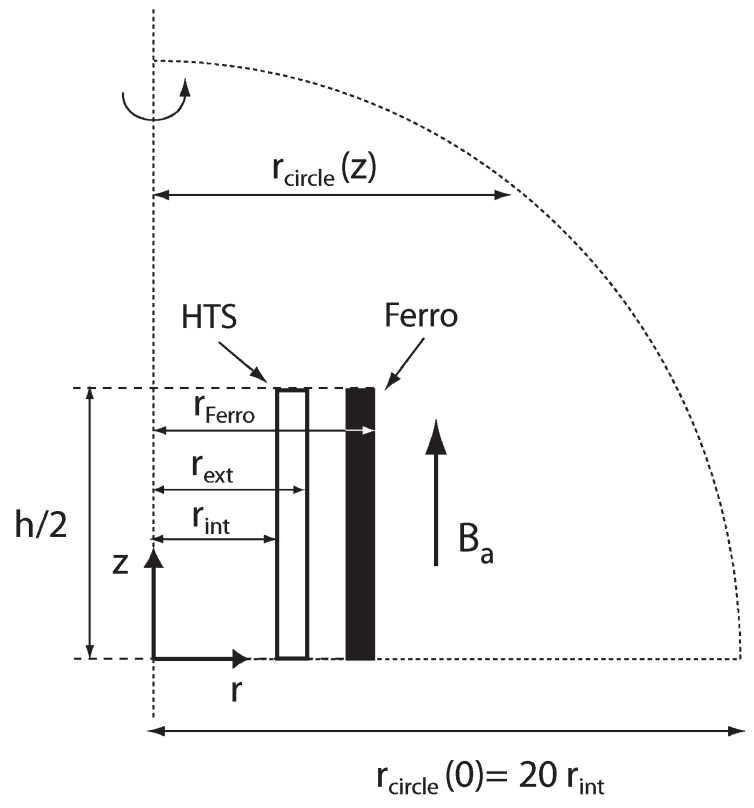

Fig. 1. Axisymmetric representation of the simulation domain. The quarter of a circle located at $r=20 r_{\text {int }}$ is not represented to scale.

magnetic flux density is given by $\mathbf{B}=\nabla \times \mathbf{A}$, and the electric field is given by $\mathbf{E}=-\partial \mathbf{A} / \partial t$. The introduction of the vector potential inside Ampere's law $\nabla \times \mathbf{H}=\mathbf{J}$ gives

$$
\nabla \times(\nu(|\mathbf{B}|) \nabla \times \mathbf{A})=-\sigma(A) \frac{\partial \mathbf{A}}{\partial t}
$$

where $\nu$ is the inverse of the magnetic permeability, with $\mathbf{H}=\nu(|\mathbf{B}|) \mathbf{B}$. Since slowly varying magnetic fields $(d B / d t<$ $1 \mathrm{~T} / \mathrm{s})$ are considered, effects associated with eddy currents induced in the ferromagnetic tube are ignored, and the conductivity of the ferromagnetic tube is taken to be 0 . The conductivity of the HTS follows a power law [28], i.e.,

$$
\sigma(A)=\frac{J_{c}(B)}{E_{c}^{1 / n}}\left|\frac{\partial \mathbf{A}}{\partial t}\right|^{\frac{1-n}{n}}
$$

where $n$ is a critical exponent, and $E_{c}$ is a critical electric field. The field-dependent critical current density is given by Kim's law [29]

$$
J_{c}(B)=\frac{J_{c 0}}{1+|\mathbf{B}| / B_{1}}
$$

where $J_{c 0}$ is the critical current density in the absence of a magnetic field, and $B_{1}$ is the critical magnetic flux density for which $J_{c}=J_{c 0} / 2$.

The HTS geometrical parameters correspond to those of a commercial $\mathrm{Bi}_{1.8} \mathrm{~Pb}_{0.2} \mathrm{Sr}_{2} \mathrm{Ca}_{2} \mathrm{Cu}_{3} \mathrm{O}_{10+x}$ (Bi-2223) tube from CAN Superconductors and are listed in Table I. The material parameters of this sample were previously extracted from measurements of the magnetic field at the center of the tube as a function of the uniform applied axial magnetic field [5], [30]; the same parameters are assumed in this study. For the HTS tube, we take $\mu_{\mathrm{HTS}}=\mu_{0}$.

The ferromagnetic material is supposed to have the magnetic properties of 1020 carbon steel, whose $B-H$ characteristic 
TABLE I

Geometrical and Material Parameters of the Bi-2223 Tube

\begin{tabular}{rcc}
\hline \hline Height & $h$ & $8 \mathrm{~cm}$ \\
External radius & $r_{\text {ext }}$ & $7.6 \mathrm{~mm}$ \\
Internal radius & $r_{\text {int }}$ & $6 \mathrm{~mm}$ \\
\hline Critical exponent & $n$ & 25 \\
Critical current density & $J_{c 0}$ & $2 \times 10^{7} \mathrm{~A} / \mathrm{m}^{2}$ \\
Critical electric field & $E_{c}$ & $10^{-4} \mathrm{~V} / \mathrm{m}$ \\
Kim's critical flux density & $B_{1}$ & $5 \mathrm{mT}$ \\
\hline \hline
\end{tabular}

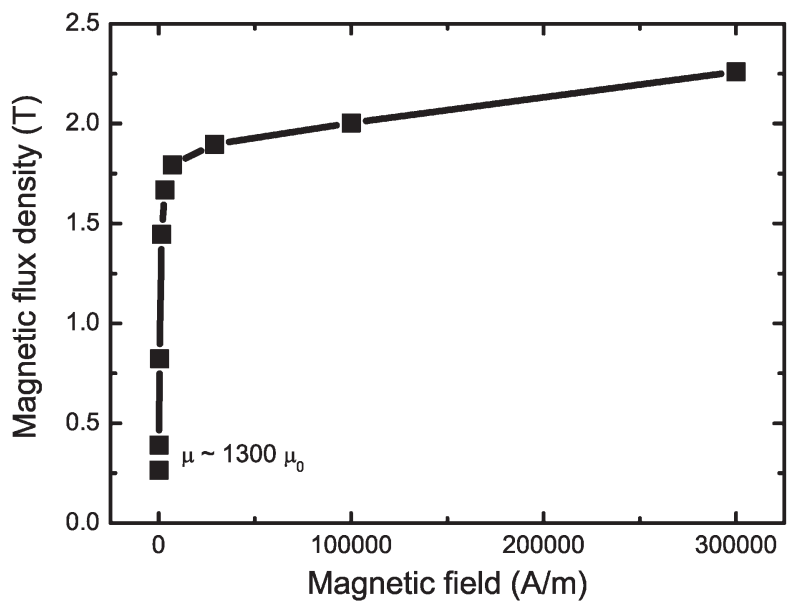

Fig. 2. $B-H$ characteristics of the ferromagnetic tube (carbon steel 1020).

may be found in [31] and is reproduced in Fig. 2. The soft ferromagnetic material is assumed to have a reversible behavior, with no hysteresis. Because the number of experimental points in [31] was not sufficient to ensure good convergence during the simulation, a Langevin equation [32]

$$
B=\mu_{0}\left\{M_{0}\left(\operatorname{coth}(a H)-\frac{1}{a H}\right)+H\right\}
$$

was first fitted to data, yielding $M_{0}=1.5 \times 10^{6} \mathrm{~A} / \mathrm{m}$ and $a=2.7 \times 10^{-3}(\mathrm{~A} / \mathrm{m})^{-1}$. Next, the inverse of the permeability $\nu(B)=B / H$ was tabulated and interpolated. This procedure yielded a low-field relative permeability of $\sim 1300$.

Boundary conditions are imposed on the external contours of the simulation domain (see Fig. 1). On the axis, $A=0$ for symmetry reasons. We force the in-plane component of $\mathbf{B}$ to vanish in the median plane for the same reasons. Finally, on the circle, we define a uniform magnetic flux density $B_{a}$ applied parallel to the tube axis, and we set $A=\left(r_{\text {circle }}(z) / 2\right) B_{a}$. The flux density $B_{a}$ is assumed to be applied as a ramp with a constant sweep rate $B_{a}(t)=\dot{B}_{a} t$, where $\dot{B}_{a}$ is fixed at $1 \mathrm{mT} / \mathrm{s}$.

The model is completely defined by (1), which is associated with the constitutive laws of (2)-(4) and subjected to the preceding boundary conditions. In order to solve our model, a FEM is used on a 2-D meshing of the simulation domain shown in Fig. 1. The numerical computation of the solution is realized within the environment of the GetDP software [26], [27]. We use an implicit Euler backward method for the temporal iterations. At each time step, a fixed-point iteration loop deals with the nonlinear terms. Details of the numerical procedure can be found in [33].
The time step choice results from a compromise between the accuracy of the simulation and a reasonable rate of convergence. We empirically set the time step for the temporal iterations at $1 \mathrm{~s}$, so that the applied magnetic flux density increases by $1 \mathrm{mT}$ between two consecutive steps. We found that smaller time steps required excessive simulation time due to the highly nonlinear magnetic permeability of the magnetic material, whereas accuracy was lost with larger time steps.

\section{Modeling the Shielding Properties of the SEPARATE TUbES}

The shielding ability of a tube is usually characterized by the shielding factor $S F$, which is defined as the ratio between the applied magnetic flux density $B_{a}$ and the magnetic flux density calculated at the center of the tube $B_{\text {in }}$, i.e.,

$$
S F\left(B_{a}\right)=\frac{B_{a}}{B_{\text {in }}} .
$$

It is also useful to define a shielding limit $B_{\text {lim }}$ that corresponds to the applied magnetic flux density below which the shielding factor exceeds 100 , i.e.,

$$
B_{\lim } \quad \text { such that } S F\left(B_{\lim }\right)=100 .
$$

With this definition, the tube is an effective shield as long as $B_{a}<B_{\text {lim }}$ and is penetrated by the external field when $B_{a}>B_{\lim }$. The choice of the factor 100 is arbitrary but is representative of a high field attenuation for $B_{a}<B_{\text {lim }}$. Other studies adopted the more stringent criterion of $S F=1000$ [5], [30].

We now turn to the shielding properties of the ferromagnetic and superconducting tubes when used separately. The calculated magnetic flux densities at the center of the Bi-2223 tube and of a ferromagnetic tube are plotted in Fig. 3(a) and (b), respectively, as a function of the applied magnetic flux density, which ranges between 0 and $40 \mathrm{mT}$. The parameters of the HTS tube are those listed in Table I. The ferromagnetic tube has a height of $h_{\text {Ferro }}=80 \mathrm{~mm}$, a wall thickness of $w=1 \mathrm{~mm}$, and an external radius of $r_{\text {Ferro }}=8.6 \mathrm{~mm}$ such that the HTS tube having the same height $h$ can be inserted inside the ferromagnetic tube.

In the HTS tube, $B_{\mathrm{in}}$ is found to be negligible for $B_{a}<$ $14 \mathrm{mT}$. At $B_{a}=14 \mathrm{mT}$, the $B_{\mathrm{in}}\left(B_{a}\right)$ curve exhibits a welldefined kink, and $B_{\text {in }}$ increases with the applied magnetic flux density when $B_{a}>15 \mathrm{mT}$. The inset shows the shielding factor in a small interval around $B_{a}=14 \mathrm{mT}$. The shielding limit $(S F=100)$ is found to be $B_{\lim }=14.04 \mathrm{mT}$; this value is consistent with the measurements and simulations of [5] and [30].

In the ferromagnetic tube, the magnetic flux density almost linearly increases with the applied flux density when $B_{a}<15 \mathrm{mT}$. The corresponding shielding factor [shown in the inset of Fig. 3(b)] is always smaller than 100, which is the threshold used for defining $B_{\text {lim }}$. From the results plotted in Fig. 3, it can be concluded that a ferromagnetic tube is a less efficient shield than an HTS tube in the axial configuration.

We checked that the simulation results are consistent with the approximate analytic expressions for the shielding factor of a 

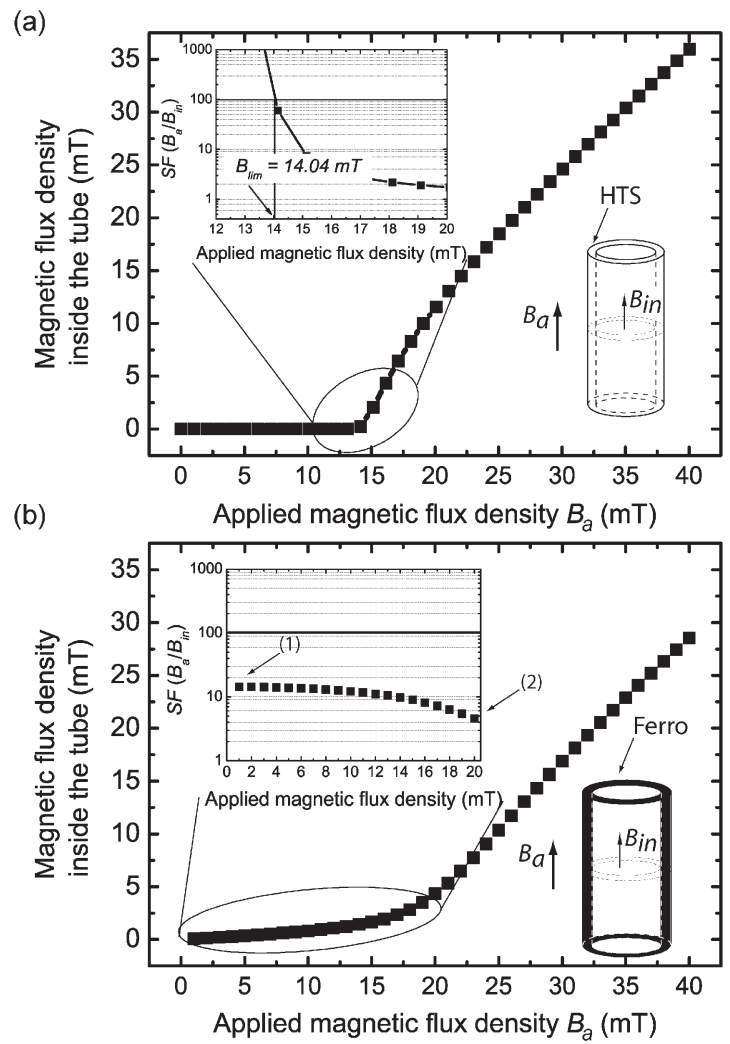

Fig. 3. Magnetic flux density calculated at the center of (a) the Bi-2223 tube and (b) a ferromagnetic tube, as a function of the applied magnetic flux density. The insets show the shielding factor $S F$ defined as $S F=B_{a} / B_{\text {in }}$. The ferromagnetic tube has a height of $80 \mathrm{~mm}$, a wall thickness of $w=1 \mathrm{~mm}$, and an external radius of $r_{\text {Ferro }}=8.6 \mathrm{~mm}$.

ferromagnetic tube [34], [35]. When a magnetic field is applied parallel to the tube axis, the shielding factor of a ferromagnetic tube of relative permeability $\mu_{r}$ is given by

$$
S F_{\text {Ferro }} \sim 4 N \frac{\mu_{r} w}{D}+1 .
$$

Here, $w$ is the thickness of the ferromagnetic layer and is assumed to be much smaller than the external diameter $D$ (i.e., $w \ll D$ ). The symbol $N$ stands for the demagnetizing factor for an ellipsoid, i.e.,

$$
N=\frac{1}{p^{2}-1}\left(\frac{p}{\sqrt{p^{2}-1}} \ln \left(p+\sqrt{p^{2}-1}\right)-1\right)
$$

where $p$ is the dimensional ratio $p=h / D$ (where $h$ is the height of the tube). Because of the nonlinear properties of the ferromagnetic material, the relative permeability $\mu_{r}$ varies with the applied magnetic field. Consider two distinct situations, with $B_{a}=1 \mathrm{mT}$ and $B_{a}=20 \mathrm{mT}$. From the simulation results presented in Fig. 3, the magnetic flux density in the ferromagnetic tube (data not shown) is found to be equal to $94 \mathrm{mT}$ for $B_{a}=1 \mathrm{mT}\left(1.69 \mathrm{~T}\right.$ for $\left.B_{a}=20 \mathrm{mT}\right)$. The resulting relative magnetic permeability is $\mu_{r}=1340\left(\mu_{r}=374\right)$. These values yield a shielding factor of $S F=20.2(S F=6.36)$, whereas the simulations give a shielding factor of $S F=14.3$ $(S F=4.6)$. Given the level of approximation used in both the simulations and the semi-analytical calculations, the agreement is satisfactory. (a)

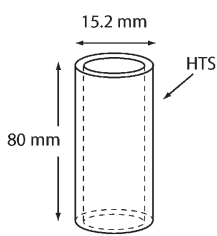

(1) - No Ferro

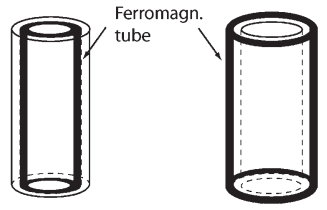

(2) - Ferro In
(3) - Ferro Out (b)

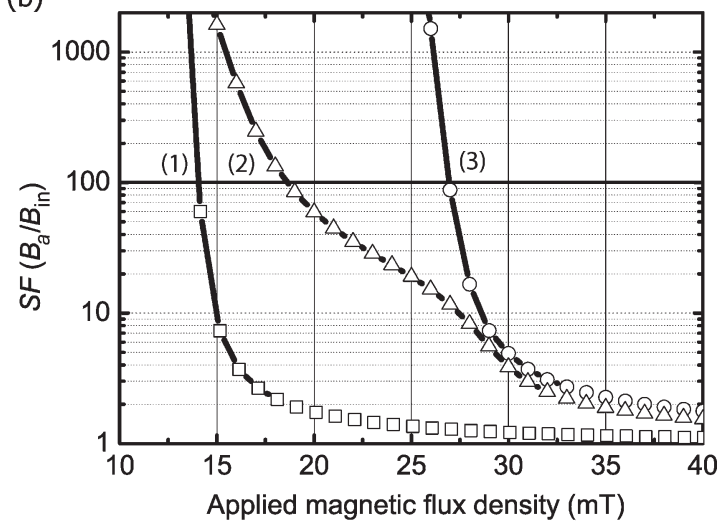

Fig. 4. (a) Configurations of the F/S tubes under consideration. The ferromagnetic tube (black) is placed either (2) inside (Ferro In) or (3) outside (Ferro Out) (1) the HTS tube. (b) Shielding factor $S F$ as a function of the applied magnetic flux density for the configurations: (1) No Ferro, (2) Ferro In, and (3) Ferro Out.

It should finally be noted that, at large magnetic flux densities (e.g., $B_{a}>20 \mathrm{mT}$ ), the shielding factor of the ferromagnetic tube is quite high, despite the saturation of the ferromagnetic layer.

\section{Shielding Properties of Hybrid Structures}

\section{A. Influence of the Position of the Ferromagnetic Tube}

We now consider the superposition of an HTS and a ferromagnetic tube in view of enhancing the shielding properties of each separate structure. We first study the differences between a ferromagnetic tube of a given height $(8 \mathrm{~cm})$ and thickness $(1 \mathrm{~mm})$ that is placed inside (Ferro In) or outside (Ferro Out) the HTS tube. Fig. 4 shows the shielding factor as a function of the applied field in the following: 1) the Bi-2223 tube alone, 2) the Ferro-In configuration, and 3) the Ferro-Out configuration. In the last two configurations, the dimensions of the ferromagnetic tube are adjusted, so that there is no air gap between the ferromagnetic and HTS tubes.

As a first observation, the shielding limits of the F/S hybrid structures exceed those of the HTS tube alone $\left(B_{\lim }=\right.$ $14.04 \mathrm{mT}$ ): In the Ferro-In configuration, $B_{\lim }$ reaches $18.7 \mathrm{mT}$, whereas it increases up to $27 \mathrm{mT}$ in the Ferro-Out configuration. Interestingly, in this latter configuration, the ferromagnetic layer turns out to be already saturated at $B_{a}=B_{\mathrm{lim}}$. Despite this, the saturation does not prevent the hybrid structure from having better shielding properties than the HTS tube alone. Saturation in the Ferro-In configuration occurs at $B_{a} \sim 27 \mathrm{mT}$, where the shielding curve in Fig. 4 shows a kink.

We now turn to analyze the two shielding configurations in greater detail. 
(a)

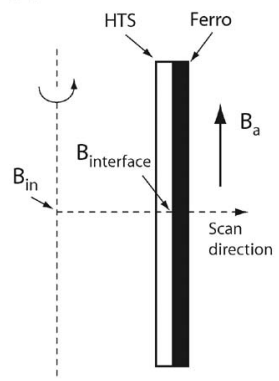

(b)

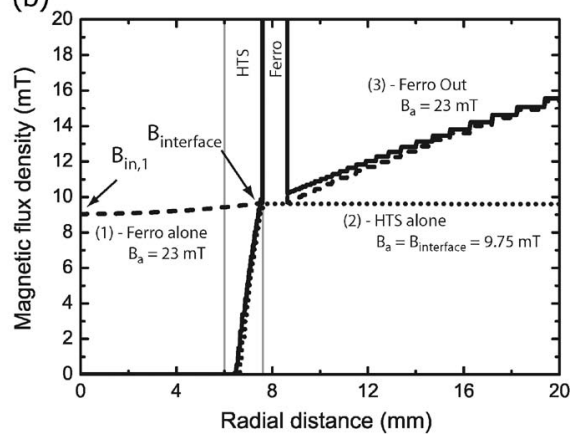

Fig. 5. (a) Axisymmetric geometry of the Ferro-Out configuration. $B_{\text {in }}$ corresponds to the magnetic flux density calculated at the center of the tube, and $B_{\text {interface }}$ is the magnetic flux density calculated at the interface between the ferromagnetic and HTS tubes. (b) Magnetic flux density profile ( $z$-component) in the median plane of the ferromagnetic tube for $B_{a}=23 \mathrm{mT}$ (dashed line) and of the HTS tube alone (dotted line) for $B_{a}=9.75 \mathrm{mT}$ (see text) and for the Ferro-Out structure with $B_{a}=23 \mathrm{mT}$.

1) Ferro-Out Configuration: As previously found, the Ferro-Out configuration yields the largest increase in the shielding limit. In fact, the shielding factor of the Ferro-Out structure can be decomposed as a combination of the shielding properties of each tube separately considered. To understand this decomposition, consider Fig. 5, which shows the profile of the $z$-component of the magnetic flux density in the median plane for three different cases: 1) only the outer ferromagnetic tube is considered as active (dashed line); 2) only the HTS tube is considered active (dotted line); and 3 ) both layers are active (solid line).

The latter case is shown with an external flux density $B_{a}=23 \mathrm{mT}$, which corresponds to a value slightly below the shielding limit. The solid line in Fig. 5 shows a decrease in the flux density as one approaches the outer surface of the ferromagnetic layer. It then abruptly increases to a high value inside the layer (in fact, the magnetic flux density there is out of the range of the graph), abruptly decreases again at the interface with the HTS layer, and, finally, rapidly decreases in the HTS layer. The abrupt jumps of $B_{z}$ across the surfaces of the ferromagnetic layer stem from the boundary conditions, which impose the continuity of the $z$-component of the magnetic field $H_{z}$. Given the high magnetic permeability of the ferromagnetic layer, the resulting flux density $B_{z}$ undergoes strong discontinuity across the boundaries.

To proceed with the analysis of the shielding properties, consider case 1 , where the ferromagnetic layer is the only active layer (dashed line in Fig. 5). The applied field is $B_{a}=23 \mathrm{mT}$. Outside the system and inside the ferromagnetic layer, the flux density profile is similar to that of case 3 . In fact, the decrease in $B_{z}$ with radius outside the ferromagnetic layer is to be attributed to demagnetizing effects: As the ferromagnetic layer canalizes the external flux lines, their density is reduced directly outside the shield. Moving to smaller radii, the flux density undergoes two abrupt jumps across the boundaries of the ferromagnetic layer and reaches a value $B_{\text {interface }}=9.75 \mathrm{mT}$ at the inner wall of the tube. At still smaller radii, the flux profile smoothly decreases to reach a center flux density $B_{\mathrm{in}, 1}=9 \mathrm{mT}$.

A comparison of the dashed and solid lines in Fig. 5 shows that the inner HTS layer plays a limited role on the flux profile

outside the ferromagnetic layer in the Ferro-Out structure. As a first approximation, the HTS layer can thus be neglected when considering magnetic fields in this region, and the analysis of the previous paragraph can be used for the Ferro-Out configuration as well.

Consider case 2, where the HTS is the only layer to act. In the spirit of decomposing the effect of the full configuration as the combined action of each layer, we consider here that the HTS layer is subjected to the field "delivered" by the ferromagnetic layer $B_{\text {interface. Several observations can be made on the flux }}$ profile [Fig. 5 (dotted line)]. First, the field profile outside the HTS layer is nearly constant; this confirms the negligible influence that the HTS layer has on its surrounding region. Second, the flux profile rapidly decreases in the HTS layer in a manner that is similar to that observed in case 3 .

To summarize, the shielding mechanism of a Ferro-Out configuration can be decomposed into two processes.

1) The ferromagnetic layer concentrates part of the applied field lines inside its bulk. As a result, the magnetic flux density directly outside the composite shield decreases with the radius. The ferromagnetic layer then delivers a resulting field $B_{\text {interface }}$ to the HTS layer.

2) The HTS layer screens its "applied field," and $B_{z}$ rapidly decreases with the radius inside the HTS wall. The resulting field $B_{\text {in }}$ permeates the inner cavity. If the "applied field" $B_{\text {interface }}$ is too weak, i.e., below the shielding limit of the HTS layer, shielding is perfect, and $B_{\text {in }}$ is zero. Thus, the condition for having perfect shielding is $B_{\text {interface }}<B_{\text {lim,HTS }}$.

The shielding factor can be written as

$$
S F_{\text {Ferro Out }}\left(B_{a}\right)=\frac{B_{a}}{B_{\text {in }}}=\frac{B_{a}}{B_{\text {interface }}} \frac{B_{\text {interface }}}{B_{\text {in }}}
$$

where the second ratio can be related to the shielding factor of the HTS layer, i.e., $B_{\text {interface }} / B_{\text {in }}=S F_{\mathrm{HTS}}\left(B_{\text {interface }}\right)$. Given that, when the ferromagnetic layer is considered to be the only active layer, the flux density smoothly varies inside the ferromagnetic tube [see Fig. 5 (dashed line)], the first factor on the right side of (9) can be approximated as $B_{a} / B_{\text {interface }} \approx$ $S F_{\mathrm{Ferro}}\left(B_{a}\right)$. As a result, the shielding factor of the hybrid structure can approximated as

$$
S F_{\text {Ferro Out }}\left(B_{a}\right) \approx S F_{\text {Ferro }}\left(B_{a}\right) S F_{\text {HTS }}\left(B_{\text {interface }}\right)
$$

which is the announced decomposition. This approximate expression is represented as a function of $B_{a}$ in Fig. 6 (open circles), together with the full shielding factor obtained by numerical simulations (filled squares). The agreement is very good.

The aforementioned results confirm the experimental relationship found by Itoh et al. [23], [24] between the shielding limit of the HTS tube alone and that obtained with the hybrid structure. These authors showed that the shielding limit of the Ferro-Out configuration is approximately equal to the shielding limit of the HTS tube alone multiplied by the shielding factor of the ferromagnetic tube. In fact, at the shielding limit of the Ferro-Out configuration, one finds $B_{\text {interface }} \approx B_{\text {lim,HTS }}$. Therefore, we have $B_{\lim }=\left(B_{\lim } / B_{\lim , \mathrm{HTS}}\right) B_{\mathrm{lim}, \mathrm{HTS}}$, where, 


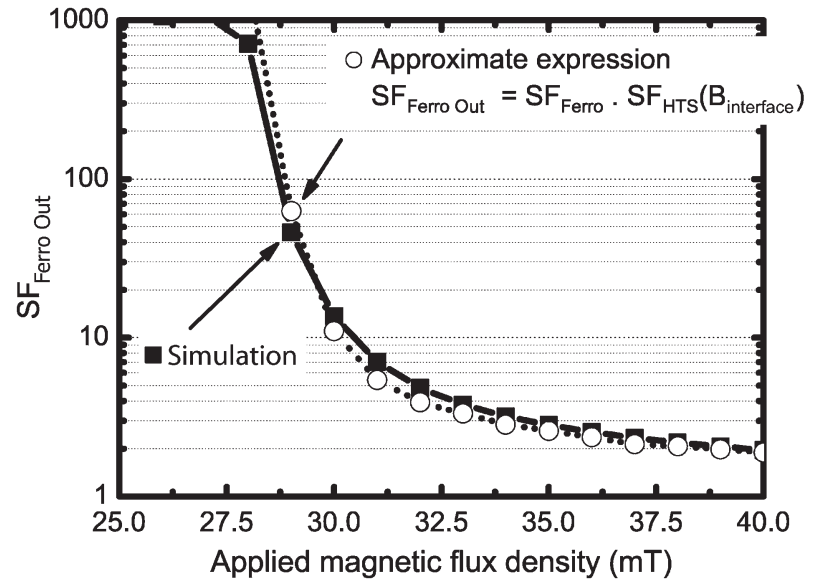

Fig. 6. Shielding factor $S F$ as a function of the applied magnetic flux density, which was calculated in the Ferro-Out structure (filled squares) and approximated [see (10)] by the product of the shielding factor of the tubes considered separately (open circles).

according to the discussion preceding $(10), B_{\lim } / B_{\lim , \mathrm{HTS}} \approx$ $B_{\lim } / B_{\text {interface }} \approx S F_{\text {Ferro }}\left(B_{\lim }\right)$. The shielding limit of the Ferro-Out configuration is thus indeed approximately given by

$$
B_{\lim } \approx S F_{\mathrm{Ferro}}\left(B_{\lim }\right) B_{\mathrm{lim}, \mathrm{HTS}}
$$

as observed in [23] and [24].

Simulation results give a shielding factor $S F_{\text {Ferro }}=2$ for an applied field such that $B_{\text {interface }}=B_{\text {lim,HTS }}$. Equation (11) thus gives

$$
B_{\lim } \approx 2 \times 14.04=28 \mathrm{mT}
$$

which is an approximated value that is close to the value of $B_{\lim }=27 \mathrm{mT}$ predicted in Fig. 4. In order to use (11) on experimental data, we must know both the shielding limit of the HTS tube alone and the shielding factor of the ferromagnetic tube when $B_{\text {interface }} \approx B_{\text {in }} \approx B_{\text {lim, HTS }}$. These values can readily be obtained from the measurement of the magnetic field at the center of each tube considered separately, as a function of an external uniform field of varying magnitude.

2) Ferro-In Configuration: We now turn to the configuration where the ferromagnetic layer is put inside the HTS tube. Fig. 7 shows the profile of the magnetic flux for an external field $B_{a}=18 \mathrm{mT}$ in two cases: 1 ) The HTS layer is considered to be the only active layer, and 2) both layers are active. In the latter case, it can be observed that the flux density slowly decreases with the radius as one approaches the system from outside. Then, it rapidly decreases inside the HTS layer, undergoes abrupt jumps across the two boundaries of the ferromagnetic layer, and finally remains almost constant for the lowest radial distances.

It would be natural to try and explain this flux profile as a result of the separate actions of each layer, as we did for the Ferro-Out configuration. From the discussion in Section IV-A1, it appears that the ferromagnetic tube mostly influences its outer region (the exterior of the tube), whereas the HTS tube mostly influences its inner region (the hollow of the tube). Because of the respective order of the tubes in the Ferro-In configuration, a higher interaction between the two layers is to be expected. (a)

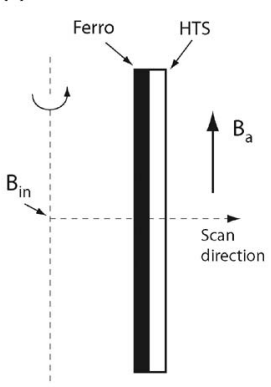

(b)

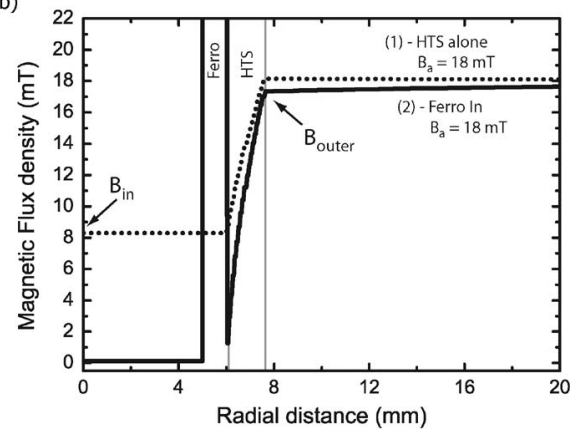

Fig. 7. (a) Axisymmetric geometry of the Ferro-In configuration. (b) Magnetic flux density profile ( $z$-component) in the median plane of the Ferro-In structure (solid line) and of the HTS tube alone (dotted line) with $B_{a}=18 \mathrm{mT}$.

Consider the differences between the solid and dotted curves in Fig. 7: When only the HTS layer is active, the flux density is almost constant outside the layer, whereas it slightly decreases in the presence of the ferromagnetic layer. If one defines $B_{\text {outer }}$ as the flux density at the outer surface of the HTS layer, one finds that $B_{\text {outer }} \sim 18 \mathrm{mT}$ for the dotted line and $B_{\text {outer }} \sim$ $17 \mathrm{mT}$ for the solid line. The drop in $B_{\text {outer }}$ is accompanied by a larger decay of the flux density inside the HTS wall when both layers are active: The drop of $\sim 1 \mathrm{mT}$ at the outer boundary of the HTS layer yields a drop of $\sim 7 \mathrm{mT}$ at the inner boundary of that layer. In fact, a faster decay rate of the magnetic flux is expected since the reduced applied field results, via the $J_{c}(B)$ law, in larger current densities and larger flux density gradients. However, the observed drop cannot entirely be accounted for by the $J_{c}(B)$ law; another mechanism involving the ferromagnetic layer is at play. The ferromagnetic layer, by canalizing part of the flux lines in its bulk, depletes the magnetic flux in the HTS layer. A straightforward decomposition of the shielding factor does not seem possible here.

\section{B. Influence of the Height of the Ferromagnetic Tube}

In the preceding analysis, the height of the ferromagnetic tube $h_{\text {Ferro }}$ was taken the same as that of the HTS tube $h_{\mathrm{HTS}}=$ $8 \mathrm{~cm}$. We now turn to study the influence of the height $h_{\text {Ferro }}$ on the shielding properties of the Ferro-In/Ferro-Out hybrid structures. The thickness of the ferromagnetic layer remains set at $w=1 \mathrm{~mm}$.

The shielding limits $B_{\lim }$ of the Ferro-Out and Ferro-In hybrid structures are plotted in Fig. 8(a) and (b), respectively, as a function of the height of the ferromagnetic tube, which varies from 8 to $160 \mathrm{~mm}$. (The height $h_{\mathrm{HTS}}=80 \mathrm{~mm}$ of the Bi-2223 tube is marked by a vertical line.) The shielding limits are calculated with the FEM simulations following the criterion $S F=100$.

1) Ferro-Out Configuration: The shielding limit in the Ferro-Out configuration exhibits a maximum for $h_{\text {Ferro }}=$ $32 \mathrm{~mm}$. The largest shielding limit is about twice as large as that obtained for cylinders of equal heights $h_{\text {Ferro }}=h_{\text {HTS }}$.

The decay of the shielding limit for $h_{\text {Ferro }}>32 \mathrm{~mm}$ can be attributed to the variation with $h_{\text {Ferro }}$ of the demagnetizing effects associated with the ferromagnetic tube. According to 
(a)

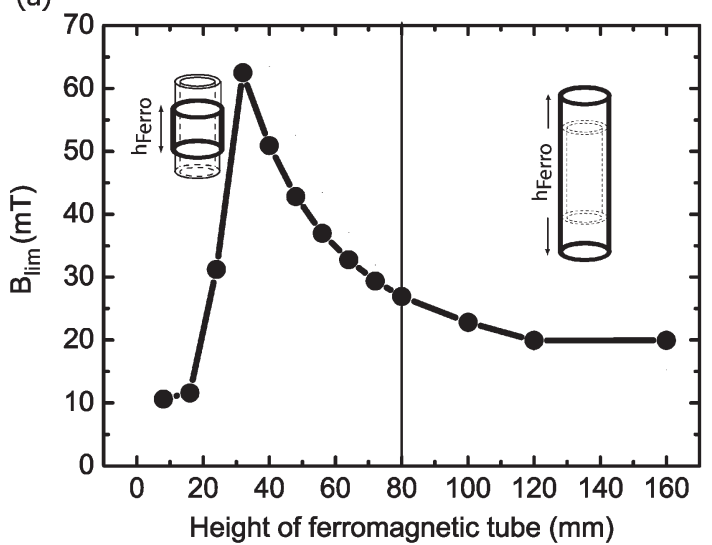

(b)

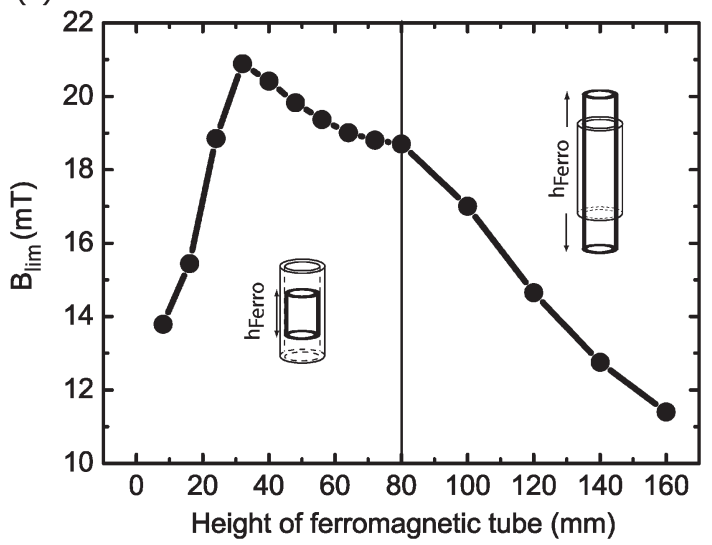

Fig. 8. Shielding limit $B_{\lim }$ as a function of the height of the ferromagnetic tube in the (a) Ferro-Out and (b) Ferro-In configurations. The HTS tube has a height of $80 \mathrm{~mm}$, which is indicated by a vertical solid line.

(7)-(11), the shielding limit in a Ferro-Out configuration may be written as

$$
B_{\lim } \approx\left(4 N\left(\frac{h_{\text {Ferro }}}{D}\right) \frac{\mu_{r} w}{D}+1\right) B_{\lim , \text { HTS }}
$$

where the demagnetizing factor $N\left(h_{\mathrm{Ferro}} / D\right)$ is given by (8). Thus, as $h_{\text {Ferro }}$ increases, both $N\left(h_{\text {Ferro }} / D\right)$ and $B_{\text {lim }}$ decrease.

For heights smaller than $32 \mathrm{~mm}$, the shielding limit rapidly decreases with $h_{\mathrm{Ferro}}$. The behavior of $B_{\lim }$ in this region is not described by (13), because this relation was established by assuming that the flux density is nearly constant inside the hollow of the structure (at the median plane). This is no longer true for the shortest heights $h_{\text {Ferro. }}$. In fact, the magnetic flux density turns out to be larger along the axis than against the inner wall of the HTS tube, because the short ferromagnetic layer fails to concentrate the magnetic field lines that are near the axis. This behavior invalidates the approximate expression established in (13).

2) Ferro-In Configuration: Fig. 8 shows a similar variation of $B_{\lim }$ as a function of $h_{\mathrm{Ferro}}$ in the Ferro-In configuration. The shielding limit exhibits a maximum for $h=32 \mathrm{~mm}$. Here, the maximum shielding limit, which is observed near $h_{\text {Ferro }}=$ $32 \mathrm{~mm}$, is only $10 \%$ higher than that in the case where $h_{\text {Ferro }}=$ $h_{\mathrm{HTS}}$, and the gain is substantially smaller than that obtained in the Ferro-Out case. (a)

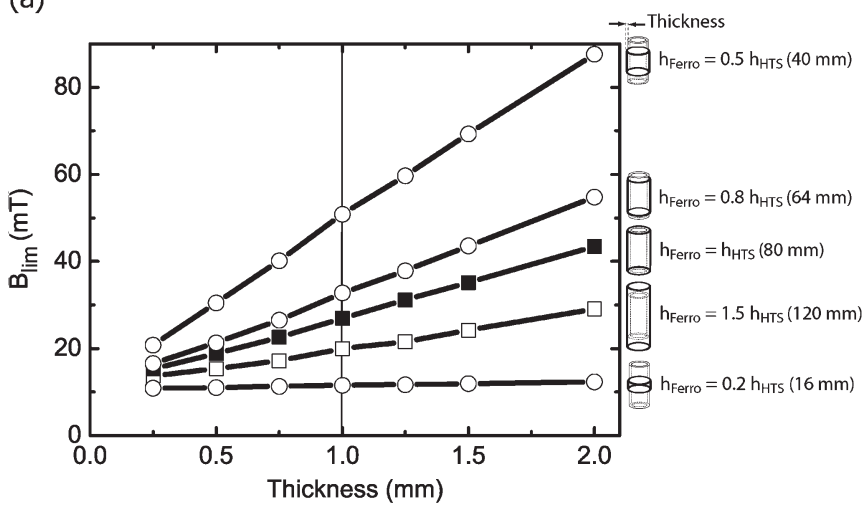

(b)

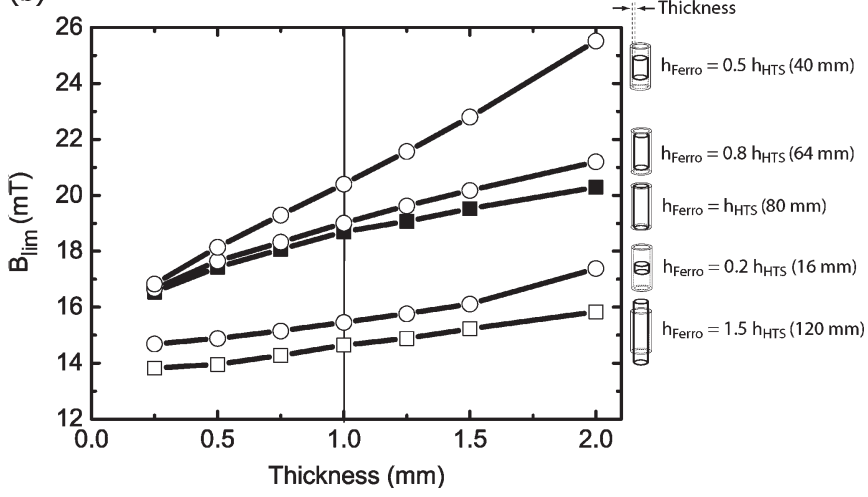

Fig. 9. Shielding limit $B_{\lim }$ as a function of the thickness of the ferromagnetic tube in the (a) Ferro-Out and (b) Ferro-In configurations. The height of the ferromagnetic tubes is $16,40,64$ (open circles), 80 (filled squares), and $120 \mathrm{~mm}$ (open squares). The vertical lines correspond to a thickness of $w=$ $1 \mathrm{~mm}$, which is the thickness of the ferromagnetic tubes in all the previous simulations.

It is interesting to note that, for both configurations, the optimal value of $h_{\text {Ferro }}$ depends on the other geometrical parameters and on the material parameters of the ferromagnetic tube.

\section{Influence of the Thickness of the Ferromagnetic Tube}

We finally consider how the thickness of the ferromagnetic tube influences the shielding limit of the F/S structure. Fig. 9(a) and (b) shows the shielding limit of the Ferro-Out and FerroIn configurations, respectively, as a function of the thickness of the ferromagnetic tube, which varies from 0.25 to $2 \mathrm{~mm}$, for a height of the ferromagnetic tube equal to $16,40,64$ (open circles), 80 (filled squares), and $120 \mathrm{~mm}$ (open squares). The shielding limit is calculated in the same manner as in Section IV-B.

In the Ferro-Out configuration, the shielding limit linearly increases with the thickness of the ferromagnetic wall for all the heights that are considered. This linear dependence is consistent with the approximate expression of (13) and with the results presented in [22]. The shielding limit can therefore be enhanced by increasing the thickness of the ferromagnetic tube.

Similarly, in the Ferro-In configuration, the shielding limit is approximately linearly dependent on the ferromagnetic tube thickness. However, the relative increase in the maximum shielding limit obtained with $h_{\text {Ferro }}=40 \mathrm{~mm}$ and $w=2 \mathrm{~mm}$ with respect to the shielding limit when $h_{\mathrm{Ferro}}=h_{\mathrm{HTS}}$ and $w=1 \mathrm{~mm}$ is only $\sim 50 \%$, which is more modest than that in the 
case of the Ferro-Out configuration, where the corresponding relative increase is $\sim 250 \%$.

\section{CONCLUSION}

We have developed a numerical model for the analysis of axisymmetric macroscopic F/S hybrid structures used as magnetic shields. The model is based on a finite-element formulation that is implemented in the open-source solver GetDP. The nonlinear electrical properties of the superconductor and the saturation in the ferromagnetic material are taken into account.

The analysis of the penetration of an applied axial magnetic field, which linearly increases with time, leads to several observations.

1) The shielding properties and, more particularly, the shielding limit of an HTS tube may substantially be improved by the addition of a ferromagnetic tube, which is placed either outside (Ferro-Out configuration) or inside (Ferro-In configuration). A Ferro-Out configuration yields the largest increase in shielding limit (Fig. 4 in Section IV).

2) The shielding effects of a Ferro-Out configuration can be decomposed into two independent processes: First, the applied magnetic field is attenuated by the ferromagnetic layer, with a factor that is nearly independent of the HTS layer. Second, the magnetic flux density decreases inside the HTS wall, according to its own shielding factor, and almost vanishes if $B_{a}<B_{\text {lim }}$. This analysis leads us to write down an approximate expression for the shielding limit that reproduces well the numerical results and can therefore be conveniently used for designing a Ferro-Out structure with known material parameters for each tube.

3) In a Ferro-In configuration, the ferromagnetic tube improves the magnetic field decay in the HTS wall. As a result, the shielding limit is enhanced (Fig. 7 in Section IV). The shielding mechanism in a Ferro-In configuration seems to involve an intricate interplay between the ferromagnetic and HTS layers, so that one must resort to numerical modeling.

4) The height of the ferromagnetic tube plays a crucial role. There exists an optimal height for which the shielding limit is maximized in both the Ferro-Out and Ferro-In configurations. Similarly, the thickness of the ferromagnetic layer should be as large as possible in order to increase the shielding limit.

\section{ACKNOWLEDGMENT}

The authors would like to thank J-H. Regnier for his participation in the early stages of this work and the University of Liége and the Fonds de la Recherche Scientifique (FRS-FNRS) for the equipment and travel grants.

\section{REFERENCES}

[1] M. Fauré, A. I. Buzdin, and D. Gusakova, "On the theory of ferromagnet/ superconductor heterostructures," Phys. C, vol. 454, no. 1/2, pp. 61-69, Apr. 2007.

[2] A. I. Buzdin, "Proximity effects in superconductor-ferromagnet heterostructures," Rev. Mod. Phys., vol. 77, no. 3, pp. 935-976, Jul. 2005.
[3] J. Y. Gu, C.-Y. You, J. S. Jiang, J. Pearson, Y. B. Bazaliy, and S. D. Bader, "Magnetization-orientation dependence of the superconducting transition temperature in the ferromagnet-superconductorferromagnet system: CuNi/Nb/CuNi," Phys. Rev. Lett., vol. 89, no. 26, p. 267001 , Dec. 2002.

[4] F. Pavese, Handbook of Applied Superconductivity. Bristol, U.K.: IoP Publishing, 1998, pp. 1461-1483.

[5] S. Denis, L. Dusoulier, M. Dirickx, P. Vanderbemden, R. Cloots, M. Ausloos, and B. Vanderheyden, "Magnetic shielding properties of high-temperature superconducting tubes subjected to axial fields," Supercond. Sci. Technol., vol. 20, no. 3, pp. 192-201, Mar. 2007.

[6] S. Denis, M. Dirickx, P. Vanderbemden, M. Ausloos, and B. Vanderheyden, "Field penetration into hard type-II superconducting tubes: Effects of a cap, a non-superconducting joint, and non-uniform superconducting properties," Supercond. Sci. Technol., vol. 20, no. 5, pp. 418-427, May 2007.

[7] J. D. Jackson, Classical Electrodynamics. Hoboken, NJ: Wiley, 1998.

[8] F. Gömöry, "Improvement of the self-field critical current of a high- $\mathrm{T}_{C}$ superconducting tape by the edge cover from soft ferromagnetic material," Appl. Phys. Lett., vol. 89, no. 7, p. 072506, Aug. 2006.

[9] F. Gömöry, M. Vojenèiak, E. Pardo, and J. Šouc, "Magnetic flux penetration and $\mathrm{AC}$ loss in a composite superconducting wire with ferromagnetic parts," Supercond. Sci. Technol., vol. 22, no. 3, p. 034017, Mar. 2009.

[10] C. Gu, A. K. M. Alamgir, T. Qu, and Z. Han, "Simulation of ferromagnetic shielding to the critical current of Bi2223/Ag tape under external fields," Supercond. Sci. Technol., vol. 20, no. 3, pp. 133-137, Mar. 2007.

[11] P. Kováè, I. Hušek, T. Melišek, M. Ahoranta, J. Šouc, J. Lehtonen, and F. Gömöry, "Magnetic interaction of an iron sheath with a superconductor," Supercond. Sci. Technol., vol. 16, no. 10, pp. 1195-1201, Oct. 2003.

[12] Y. Mawatari, "Magnetic field distributions around superconducting strips on ferromagnetic substrates," Phys. Rev. B, Condens. Matter, vol. 77, no. 10, p. 104505 , Mar. 2008.

[13] M. Majoros, B. A. Glowacki, and A. Campbell, "Magnetic screening as a possible way to decrease transport AC losses in multifilamentary superconductors-Basic theoretical considerations," Phys. C, vol. 334, no. 3/4, pp. 129-140, Jun. 2000.

[14] S. H. Rupp, A. D. Caplin, and M. P. Staines, "Magnetisation AC-loss measurements on $\mathrm{YBa}_{2} \mathrm{Cu}_{3} \mathrm{O}_{7}$ tapes with weakly-ferromagnetic NiW substrates," J. Phys., Conf. Ser., vol. 97, p. 012078, 2008.

[15] N. Amemiya and M. Nakahata, "Numerical study on AC loss characteristics of superconducting power transmission cables comprising coated conductors with magnetic substrates," Phys. C, vol. 463-465, pp. 775780 , Oct. 2007.

[16] F. Grilli, S. P. Ashworth, and L. Civale, "Interaction of magnetic field and magnetic history in high-temperature superconductors," J. Appl. Phys., vol. 102 , no. 7 , p. 073909 , Oct. 2007.

[17] A. Campbell and D. Cardwell, "Bulk high-temperature superconductors for magnet applications," Cryogenics, vol. 37, no. 10, pp. 567-575, Oct. 1997.

[18] X. Granados, M. Torner, T. Puig, and X. Obradors, "Magnetization of ferromagnetic-SC heterostructures for trapped field low power motors," IEEE Trans. Appl. Supercond., vol. 17, no. 2, pp. 1629-1632, Jun. 2007.

[19] S. V. Yampolskii, Y. A. Genenko, H. Rauh, and A. V. Snezhko, "The Bean model of the critical state in a magnetically shielded superconductor filament," J. Phys., Conf. Ser., vol. 43, pp. 576-580, 2006.

[20] M. Itoh, T. Ohyama, K. Hoshino, H. Ishigaki, and T. Minemoto, "Improvement in magnetic shielding by the superposition of a magnetic cylinder over a copper-oxide superconducting cylinder," IEEE Trans. Appl. Supercond., vol. 3, no. 1, pp. 181-184, Mar. 1993.

[21] M. Itoh, K. Mori, and T. Minemoto, "Magnetic shielding effects by the superposition of a six-layered ferromagnetic cylinder over a BPSCCO cylinder," IEEE Trans. Magn., vol. 32, no. 4, pp. 2605-2608, Jul. 1996.

[22] M. Itoh, "Influence of wall thickness on magnetic shielding effects of BPSCCO cylinders," in Advances in Cryogenic Engineering-Materials, vol. 40. New York: Plenum, 1994, pp. 261-270.

[23] K. Mori, T. Minemoto, and M. Itoh, "Magnetic shielding of the superposition of a hybrid ferromagnetic cylinder over a BPSCCO cylinders," IEEE Trans. Appl. Supercond., vol. 7, no. 2, pp. 378-381, Jun. 1997.

[24] M. Itoh, T. Ohyama, K. Mori, and T. Minemoto, "Magnetic shielding effect of high- $T_{c}$ superconducting cylinder using the ferromagnetic cylinder," Proc. Inst. Elect. Eng. Jpn., vol. 115-C, pp. 696-701, 1995.

[25] E. Maher, J. S. Abell, R. I. Chakalova, Y. L. Cheung, T. W. Button, and P. Tixador, "Multi-layer coated conductor cylinders-An alternative approach to superconducting coil fabrication," Supercond. Sci. Technol., vol. 17 , no. 12 , pp. $1440-1445$, Dec. 2004 
[26] P. Dular and C. Geuzaine, GetDP Reference Manual: The Documentation for GetDP, a General Environment for the Treatment of Discrete Problems, 2006. [Online]. Available: http://www.geuz.org/getdp/

[27] P. Dular, C. Geuzaine, A. Genon, and W. Legros, "An evolutive software environment for teaching the finite element method in electromagnetism," IEEE Trans. Magn., vol. 35, no. 3, pp. 1682-1685, May 1999.

[28] J. Rhyner, "Magnetic properties and AC-losses of superconductors with power law current-voltage characteristics," Phys. C, vol. 212, no. 3/4, pp. 292-300, Jul. 1993.

[29] Y. Kim, C. Hempstead, and A. Strnad, "Flux creep in hard superconductors," Phys. Rev., vol. 131, no. 6, pp. 2486-2495, Sep. 1963.

[30] J.-F. Fagnard, M. Dirickx, M. Ausloos, G. Lousberg, B. Vanderheyden, and P. Vanderbemden, "Magnetic shielding properties of high-Tc superconducting hollow cylinders: Experimental and modelling results in axial and transverse magnetic field configurations," Supercond. Sci. Technol., vol. 22, p. $105002,2009,10$ pp.

[31] [Online]. Available: http://geuz.org/getdp/wiki/magnetostaticsnonlinear

[32] S. Blundell, Magnetism in Condensed Matter. London, U.K.: Oxford Univ. Press, 2003

[33] G. P. Lousberg, M. Ausloos, C. Geuzaine, P. Dular, P. Vanderbemden, and B. Vanderheyden, "Numerical simulation of the magnetization of hightemperature superconductors: 3D finite element method using a single time-step iteration," Supercond. Sci. Technol., vol. 22, no. 5, p. 055005, May 2009.

[34] A. Mager, "Magnetic shields," IEEE Trans. Magn., vol. MAG-6, no. 1, pp. 67-75, Mar. 1970.

[35] A. Mager, "Magnetic shielding efficiencies of cylindrical shells with axis parallel to the field," J. Appl. Phys., vol. 39, no. 3, p. 1914, Feb. 1968.

Gregory P. Lousberg was born in Liége, Belgium, in 1982. He received the M.Sc. degree from the Swiss Federal Institute of Technology (EPFL), Lausanne, Switzerland, in 2005, and the Engineer degree from the University of Liége (ULg), Liége, in 2006, both in physics. He is currently working toward the Ph.D. degree in bulk HTS superconductors under the co-supervision of Prof. Vanderheyden and Prof. Vanderbemden at the ULg.

During his engineering studies at ULg, he spent two years at EPFL (2004-2005) under the European TIME exchange program. During this period, he was working toward the Master's degree and was appointed to the Laboratory of Physics of Nanostructures of Prof. E. Kapon, where he worked on arrays of coupled vertical-cavity surface-emitting lasers. In 2006, he realized his second master thesis at the Interuniversity MicroElectronic Center (IMEC), Leuven, Belgium, where he worked on the optimization of Schottky-Barrier field-effect transistors. He is currently an FRS-FNRS Research Fellow with the SUPRATECS, Department of Electrical Engineering and Computer Science, ULg.
Jean-Francois Fagnard was born in Charleroi, Belgium, in 1977. He received the M.Sc. and M.E. degrees from the University of Liége (ULg), Liége, Belgium, in 1998 and 2000, respectively, both in physics. He is currently working toward the Ph.D. degree in magnetic shielding with HTS superconductors, under the co-supervision of Col. Dirickx from the Royal Military Academy, Brussels, Belgium, and Prof. Vanderbemden from ULg. His first master thesis dealt with photocurrent measurements on $\mathrm{ZnSe} / \mathrm{GaAs}$ heterojunctions. His second thesis was focused on diffractive optics applied to a guided mode resonance device.

$\mathrm{He}$ is currently a Royal Military Academy Researcher with the SUPRATECS, Department of Electrical Engineering and Computer Science, ULg, and also with the CISS Department, Royal Military Academy.

Marcel Ausloos received the Ph.D. degree from Temple University, Philadelphia, PA, in 1973. His thesis with K. Kawasaki was on mixing condensed matter and statistical physics, and continued application of statistical physics ideas to many problems and phenomena, ranging from thermodynamic phase transitions to various types of extreme events characterizing societies.

$\mathrm{He}$ is currently an Engineer and Director of the SUPRATECS, Department of Electrical Engineering and Computer Science, University of Liége, Liége, Belgium. He is the author of more than 500 reviewed papers in top scientific journals. He edited six books and organized many scientific meetings. His work is often mentioned in the press and other media.

Philippe Vanderbemden received the M.Sc. degree in electrical engineering and the Ph.D. degree from the University of Liége (ULg), Liége, Belgium, in 1995 and 1999, respectively.

He was a Research Fellow and Postdoctoral Researcher with the Belgian National Fund for Scientific Research (FNRS) and spent six months at the IRC in Superconductivity, Cambridge University, Cambridge, U.K. In 2003, he joined the academic staff of the ULg. He is currently a Professor in the Department of Electrical Engineering and Computer Science, ULg, where he is with the SUPRATECS research group. His research interests include magnetic and transport properties of high-temperature superconductors, and related magnetic materials geared toward engineering applications, as well as high-sensitivity electrical and magnetic measurements.

Benoît Vanderheyden (M'05) was born in Verviers, Belgium, in 1969. He received the B.E. degree from the University of Liége (ULg), Liége, Belgium, in 1992, and the Master's and Ph.D. degrees from the University of Illinois, Urbana, where he studied the quasiparticle excitations of quark-gluon plasmas under the supervision of G. Baym, in 1994 and 1998, respectively.

From 1998 to 2000, he was a Postdoctoral Fellow at the Niels Bohr Institute, Copenhagen, Denmark. In 2000, he joined the ULg, as an Assistant Professor. $\mathrm{He}$ is currently a Professor in the Department of Electrical Engineering and Computer Science, ULg, where he is with the SUPRATECS research group. His research interests are the electric and magnetic properties of materials, including the study of superconducting materials used for permanent magnets or magnetic shields, and the modeling of phase transitions in high-Tc compounds. 